Erratum

\title{
Time-domain simulation of the circular Bragg phenomenon exhibited by axially excited chiral sculptured thin films
}

J.B. Geddes III and A. Lakhtakia

CATMAS - Computational and Theoretical Materials Sciences Group, Department of Engineering Science and Mechanics, 212 Earth-Engineering Sciences Building, Pennsylvania State University, University Park, PA 16802-6812, USA

Eur. Phys. J. AP 14, 97-105 (2001)

A transcription error crept in the last term on the right sides of equations (10), (12) and (14) of the referred paper above. Please replace -1 by $-\delta(t)$. All other equations as well as our results are not affected. We regret any inconvenience caused by this error. 